\title{
1 The influence of unmeasured confounding on the MR Steiger approach
}

\author{
Sharon M. Lutz ${ }^{1,2,0]}$, Kirsten Voorhies ${ }^{1}$, Ann Chen $\mathrm{Wu}^{1}$, \\ John Hokanson ${ }^{3}$, Stijn Vansteelandt 4,5 , Christoph Lange ${ }^{2}$
}

1. PRecision Medicine Translational Research (PROMoTeR) Center, Department of Population Medicine, Harvard Medical School and Harvard Pilgrim Health Care Institute,

7 Boston, MA, USA

8 2. Department of Biostatistics, Harvard T.H. Chan School of Public Health, Boston, MA ,USA

9 3. Department of Epidemiology, University of Colorado Anschutz Medical Campus, Aurora, 10 CO, USA

11 4. Department of Applied Mathematics, Computer Science and Statistics, Ghent University, 12 Ghent, BE

13 5. Department of Medical Statistics, London School of Hygiene and Tropical Medicine,

14 London, UK

15 ? For Correspondence: Sharon Lutz PhD, Department of Population Medicine, Harvard 16 Medical School and Harvard Pilgrim Health Care Institute, 401 Park Drive, Suite 401 East,

17 Boston, MA 02215, Phone: 617-867-4959, E-mail: smlutz@hsph.harvard.edu 


\section{Abstract}

20 The Mendelian Randomization (MR) Steiger approach is used to determine the

21 direction of a possible causal effect between two phenotypes [1]. For two

22 phenotypes, denoted phenotype 1 and 2, the MR Steiger approach is composed of

23 two parts: (1) MR is performed for a set of single nucleotide polymorphisms (SNPs)

24 that serve as instrumental variables for phenotype 1 and (2) the difference of two

25 correlations, the correlation between the SNPs and phenotype 1 and the correlation

26 between the SNPs and phenotype 2, is calculated. These two parts are then used to

27 determine the direction of a possible causal effect between the two phenotypes. The

28 original MR Steiger paper [1] shows that unmeasured confounding of the two

29 phenotypes affects the validity of the MR Steiger approach, but does not elucidate as

30 to how this occurs. In particular, it was argued that if the magnitude of the

31 observational variance explained between the two phenotypes is above 0.2 , the MR

32 Steiger method may return the incorrect causal direction due to unmeasured

33 confounding. This may initially seem surprising since unmeasured confounding

34 does not induce spurious associations between the SNP and phenotype 2, as we

35 demonstrate using directed acyclic graphs. In this note, we show that this is because

36 unmeasured confounding may rescale the magnitude of a non-zero association, and

37 thereby distort the comparison of the correlation between the SNP and phenotype 2

38 and the correlation between the SNP and phenotype 1 . We will end with a number

39 of cautionary remarks on the MR Steiger method, which are partly motivated by this

40 and mentioned in the original MR Steiger paper [1]. 
41 Let $g$ denote the SNP, $x$ denote phenotype $1, y$ denote phenotype 2 , and $u$ denote

42 an unmeasured confounder of the association between both phenotypes. Then, $x$

43 and $y$ can be modeled as follows in the original MR Steiger paper [1]:

$$
\begin{aligned}
& x=\alpha_{1}+\beta_{g} g+\beta_{u 1} u+\epsilon_{1} \\
& y=\alpha_{2}+\beta_{x} x+\beta_{u 2} u+\epsilon_{2}
\end{aligned}
$$

45 The $\alpha_{\text {曰 }}$ terms represent intercepts and the terms denote random errors, which

46 are assumed to be independent and normally distributed with mean zero (given $\mathrm{g}, \mathrm{x}$

47 and $\mathrm{u}$ ). This relationship is visualized in the directed acyclic graph (DAG), Figure 1. $x$

48 is a collider on the path from $g$ to $y$ via $u$, and hence unmeasured confounding

49 between $x$ and $y$ does not induce spurious associations between $g$ and $y$ [2]. The

50 advantage of reasoning with DAGs is that the conclusion holds in a nonparametric

51 sense; that is, it holds even when the models are not linear as in equation (1).

52 However, in spite of this, unmeasured confounding may affect the magnitude of a

53 non-zero correlation between $\mathrm{g}$ and $\mathrm{y}$, and therefore also the comparison of

54 correlations between $\mathrm{g}$ and $\mathrm{x}$ versus $\mathrm{g}$ and $\mathrm{y}$. This may occur because unmeasured

55 confounding affects the phenotypic variances, and may in particular make the

56 variance of $y$ smaller than the variance of $\mathrm{x}$ as seen below. Understanding this

57 artefact of the MR Steiger approach could prove helpful for understanding and

58 utilizing the approach.

59

60 Assuming the standard regression framework and equation (1), then 


$$
\operatorname{Cor}(g, x)^{2}=\left[\frac{\operatorname{Cov}(g, x)}{\sqrt{\operatorname{Var}(g) \operatorname{Var}(x)}}\right]^{2}=\left[\frac{\operatorname{Cov}\left(g, \alpha_{1}+\beta_{g} g+\beta_{u 1} u+\epsilon_{1}\right)}{\sqrt{\operatorname{Var}(g) \operatorname{Var}(x)}}\right]^{2}=\frac{\beta_{g}^{2} \operatorname{Var}(g)}{\operatorname{Var}(x)}
$$$$
\operatorname{Cor}(g, y)^{2}=\left[\frac{\operatorname{Cov}\left(g, \alpha_{2}+\beta_{x} x+\beta_{u 2} u+\epsilon_{2}\right)}{\sqrt{\operatorname{Var}(g) \operatorname{Var}(y)}}\right]^{2}=\left[\frac{\beta_{x} \operatorname{Cov}(g, x)}{\sqrt{\operatorname{Var}(g) \operatorname{Var}(y)}}\right]^{2}=\frac{\beta_{x}^{2} \beta_{g}^{2} \operatorname{Var}(g)}{\operatorname{Var}(y)}
$$

Therefore, the covariance between $g$ and $y$ does not depend on $\beta_{u 2}$ and is thus the

64 same, regardless of whether there is unmeasured confounding. The more general

65 reasoning under the DAG in Figure 1 confirms that unmeasured confounding does

66 not induce spurious associations between $g$ and $y$. In spite of this, the comparisons

67 of the correlations may be affected by unmeasured confounding. Indeed, using equation (1), $y$ can be rewritten such that

$$
\begin{gathered}
x=\alpha_{1}+\beta_{g} g+\beta_{u 1} u+\epsilon_{1} \\
y=\left(\alpha_{2}+\beta_{x} \alpha_{1}\right)+\beta_{x} \beta_{g} g+\left(\beta_{x} \beta_{u 1}+\beta_{u 2}\right) u+\beta_{x} \epsilon_{1}+\epsilon_{2}
\end{gathered}
$$

70 If the signs of $\beta_{u 2}$ and $\beta_{x} \beta_{u 1}$ differ (i.e. $\beta_{u 2}>0$ and $\beta_{x} \beta_{u 1}<0$ or $\beta_{u 2}<0$ and $\beta_{x} \beta_{u 1}>0$ ), then

71 the unmeasured confounder increases the variance of $\mathrm{x}$ but may sometimes

72 decrease the variance of $y$. In particular, if $\operatorname{Var}(y)<\beta_{x}^{2} \operatorname{Var}(x)$ then

$73 \operatorname{Cor}(g, x)^{2}<\operatorname{Cor}(g, y)^{2}$ and the false direction may be detected. This occurs when

74 unmeasured confounding biases the effect of $\mathrm{x}$ on $\mathrm{y}$ towards the null to a sufficient

75 degree. This is an artefact of working with correlations, which do not have the

76 property that they shrink as one moves further in the causal chain. 
78 Consider the following simple simulation study. For $n=100,000$ subjects, we

79 generated the SNP $g$ and the unmeasured confounder $u$ from standard normal

80 distributions. $\mathrm{x}$ and $\mathrm{y}$ are generated from equation 1 such that the intercepts equal 0

81 (i.e. $\alpha_{1}=0$ and $\alpha_{2}=0$ ), the genetic effect size, $\beta_{g}=1$, and the effect of $x$ on $y, \beta_{x}=1$.

82 The random errors $\epsilon_{1}$ and $\epsilon_{2}$ are generated from standard normal distributions. We

83 considered $\beta_{u 1}=-5$ and vary $\beta_{u 2}$ from 0 to 12 . As seen in Figure 2 , there is a range

84 from about 0.1 to 9.5 for $\beta_{u 2}$ such that estimated $\operatorname{Cor}(g, x)<\operatorname{Cor}(g, y)$ and the MR

85 Steiger approach detects the wrong causal direction. While we considered only one

86 example here, we have created an R package that runs these simulations scenarios

87 for various parameters. The R package called UCMRS is available on GitHub

88 (https://github.com/SharonLutz/UCMRS).

89 These results call for caution in using the MR-Steiger method to infer causal

90 directions. It is indeed rarely the case that the association between 2 phenotypes is

91 unconfounded; in fact, concerns about confounding form the key motivation behind

92 MR methods. In line with the original MR Steiger manuscript [1], caution is

93 especially warranted when unmeasured confounding is expected to induce a bias in

94 a direction opposite to the effect between both phenotypes. Other major concerns

95 about the MR-Steiger method come from the assumption that $g$ has no direct effect

96 on one of the phenotypes. [1,2] Indeed, in settings where it is not a priori known

97 whether $x$ influences $y$ or $y$ influences $x$, there can be no biological basis to conclude

98 that $g$ has no direct effect on either $x$ or $y$, since assumptions about the absence of a 
99 direct effect demand knowledge as to whether $x$ influences $y$ or vice versa. This

100 makes the MR-Steiger method reliant on strong mathematical convenience

101 assumptions. Sensitivity analyses are needed to study the effects of confounding by

102 unmeasured variables and pleiotropy on the MR Steiger approach.

\section{Acknowledgements}

104 Research reported in this publication was supported by the National Institutes of

105 Health grants K01HL125858 (SML), NICHD R01HD085993 (ACW), and the Cure

106 Alzheimer's Fund (CL). We would like to thank Dr. Kate Tilling for helpful

107 discussions and for suggesting the considered simulation study example.

\section{Conflict of Interest}

109 The authors have no conflict of interest to declare.

\section{References}

111 [1] Hemani, G., Tilling, K., Davey Smith, G. Orienting the causal relationship

112 between imprecisely measured traits using GWAS summary data. PLOS Genetics.

$1132017 ; 13(11), \mathrm{e} 1007081$.

114 [2] Lutz SM, Wu AC, Hokanson JE, Vansteelandt S, Lange C. Caution against

115 examining the role of reverse causality in Mendelian Randomization. Gen Epi. 2021;

116 https://doi.org/10.1002/gepi.22385 (In Press) 
bioRxiv preprint doi: https://doi.org/10.1101/2021.10.28.466267; this version posted November 2, 2021. The copyright holder for this preprint (which was not certified by peer review) is the author/funder. All rights reserved. No reuse allowed without permission.

117 


\section{Figures}

119 Figure 1: In the DAG below, $g$ is the SNP, $x$ is phenotype 1, $y$ is phenotype 2, and $u$ is 120 the unmeasured confounder. Note that $x$ is a collider on the path from $g$ to $y$ via $u$, 121 and hence the association between $g$ and $y$ is the same regardless of whether there 122 is unmeasured confounding between $x$ and $y$.

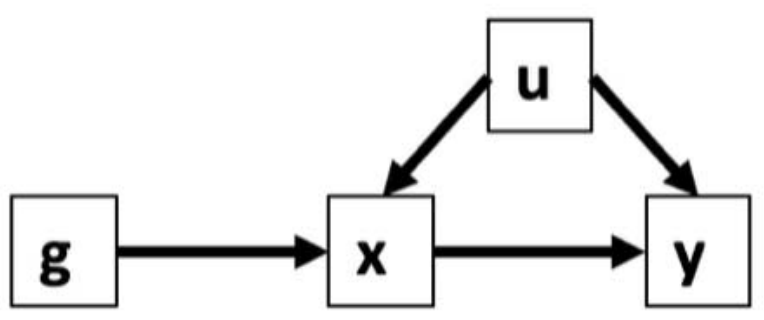


124 Figure 2: Proportion of simulations where the estimated $\operatorname{Cor}(g, x)<\operatorname{Cor}(g, y)$ and the 125 MR Steiger approach concludes the wrong direction (i.e. $y->x$ ).

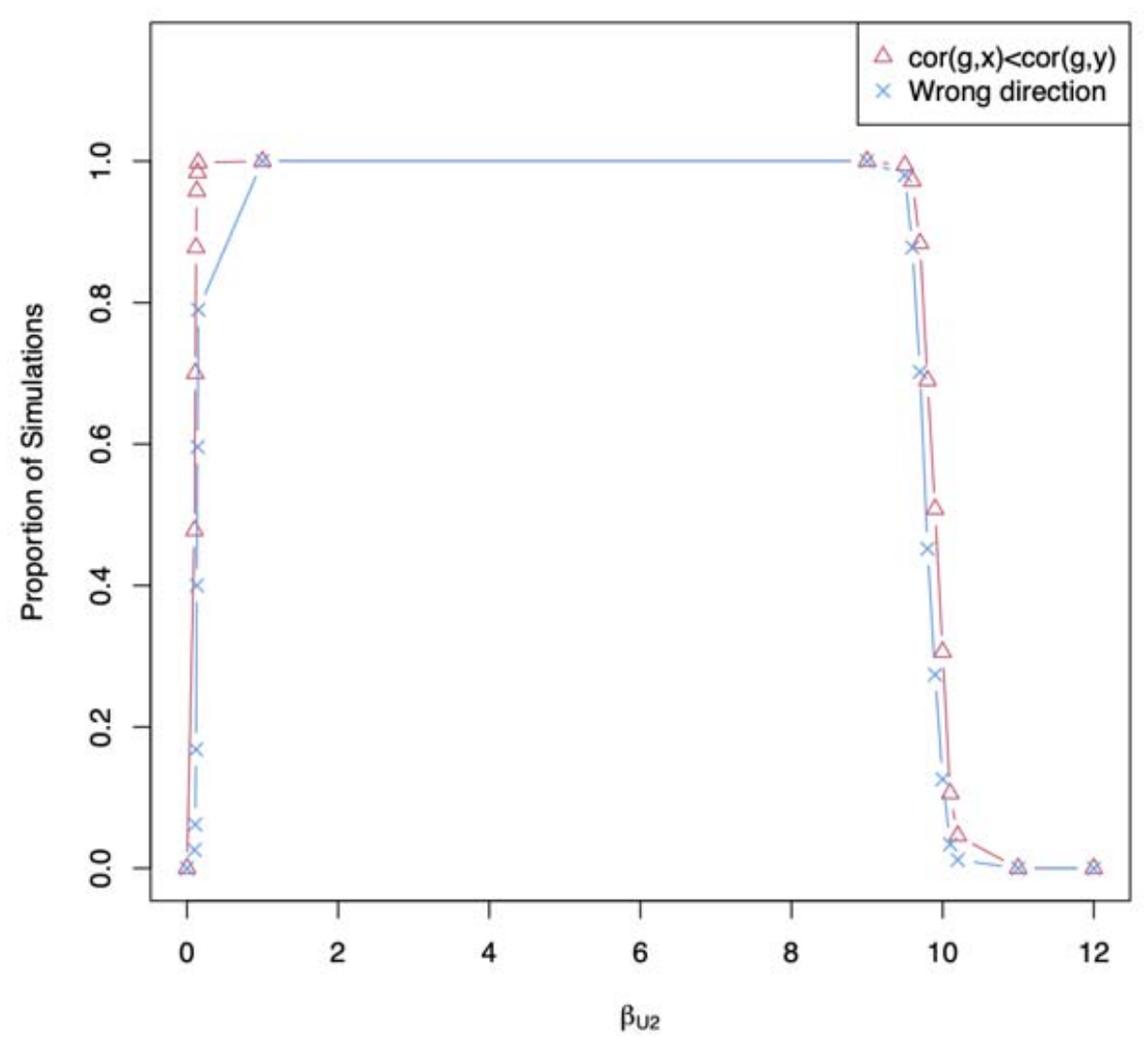

126 